\title{
Performance Test of Future Network OpenFlow Soft Switch
}

\author{
Xie Peizhang, Zhou Jungui, Wang Jingyu
}

Nanjing Institute of Product Quality Inspection, Nanjing 21012, China

Email: xpzseu@163.com

Keywords: SDN, OpenFlow Switch, Performance Test

\begin{abstract}
SDN (Software Defined Network) is the important part of Future Network, and the OpenFlow soft switch which separates the control plane from the data plane is the key instrument. While there is less research on the performance model of SDN, and also less research on experimental methods of performance test of future network. In this paper, in view of the current major performance defects of the model design, some test methods are designed to find out the impact of various factors on the performance of SDN, it is the basic experiment for establish the performance model. In this paper, the OpenFlow switch are analyzed, and the main performance parameters are throughput, packet loss and time-delay, the influence factors are load, packet size and the transforming packet quantity, the test expriment overcome the disadvantages of less parameters of current SDN performance model. In particular, the transmission packet quantity test method is designed, and influence of the transmission packet quantity is proposed in this paper firstly.
\end{abstract}

\section{Introduction}

With the rapid development of cloud computing, big data, internet of things and mobile internet, in order to overcome the shortcomings of the existing network structure, the future network arises; SDN (software defined network) is an important development direction of the future network. SDN aims at realizing the definition of network interconnection and network behavior and the open interface, which can support the innovation of new network architecture and new business. OpenFlow as the one of the realization of $\mathrm{SDN}^{[1]}$, central control node driven by software automatic control the entire network, simplify the network configuration mode, increase the openness of the network, and has become the foundation of the GENI, FINE and other countries future network projects ${ }^{[2]}$.

OpenFlow system mainly contains OpenFlow switch and controller. The switch is responsible for data forwarding, contains the flow table, the secure channel and the OpenFlow protocol, such as Fig.1. In the controller, the Network Operating System realize the control logic function, NOX is the OpenFlow network central executive unit to realize the programmable control. The OpenFlow network based on NOX, NOX is the control core, openflow maintains the basic information of the whole network by the network view, applications running on the NOX by invoking the network view of global data and operation of openflow switches to manage and control the whole network.

OpenFlow switch is the key device of small test network and the manage-control separation architecture, and performance of the switch is one of the key factors to evaluate the network. However, most of the current research on OpenFlow mainly focus on the following parts,(1)The design problem of the forwarding plane ${ }^{[3]}$;(2) scalability of control plane ${ }^{[4]}$;(3)The consistency of control logic of $\mathrm{SDN}^{[5]}$;(4)Research on how to improve the performance of data plane ${ }^{[6]}$;(5)Model of OpenFlow.In paper [7],Based on measurements of switching times of current OpenFlow hardware, a basic model for the forwarding speed and blocking probability of an OpenFlow switch is proposed. In paper [8], CPN (colored Petri net) is used to analyze and test OpenFlow. However there is less research on the performance model of OpenFlow soft switch. 


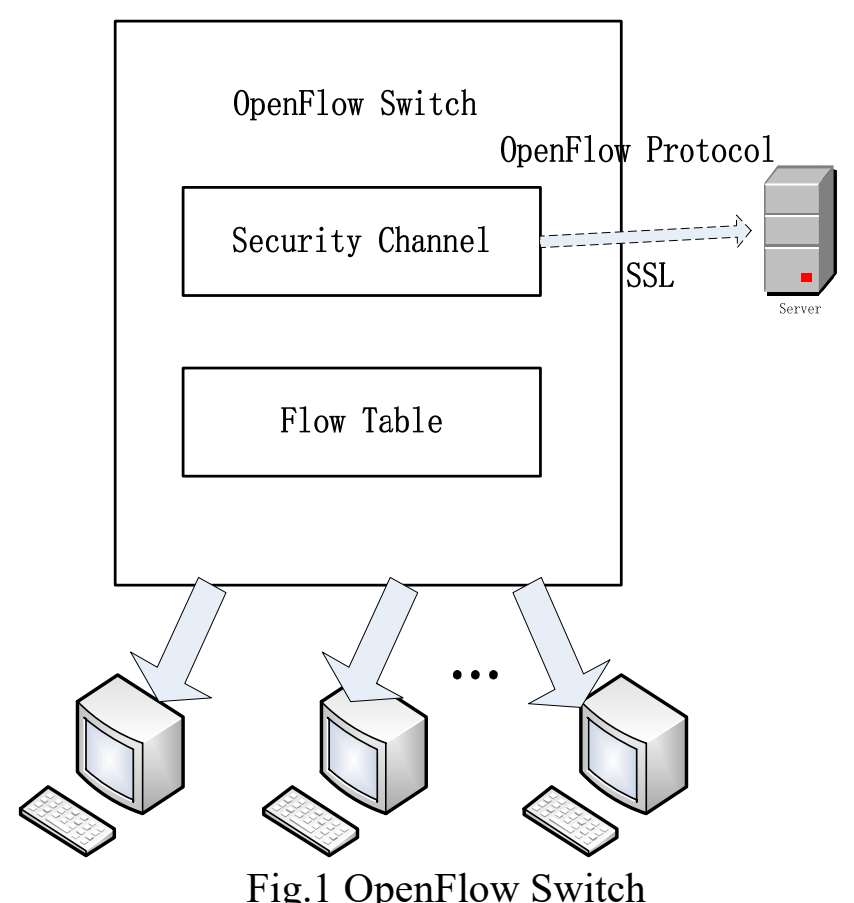

In this paper, the OpenFlow switch is analyzed; the study shows the performance parameters of the SDN are load, packet size and the transforming packet quantity.

\section{Working Principle of OpenFlow Switch}

The processing unit of the OpenFlow switch is composed of the flow table. Each flow table is composed of a number of flow table items; the flow table item represents the forwarding rule of the OpenFlow network, data packets entering the switch to obtain the corresponding operation through the query flow table [9]. In order to improve the query efficiency, the current flow table is obtained by using the multi-level flow table and assembly line mode, and then the operation can be obtained by the flow table. Flow table is mainly composed of Match Field, Counter and Instruction. The structure of the match field contains many matching items, which covers most of the link layer, network layer and transport layer. With the continuous update of the OpenFlow protocol, VLAN, MPLS and IPV6 protocols are also extended to the OpenFlow standard. Because the OpenFlow switch is matching and forwarding mode, the OpenFlow network will no longer distinguish between routers and switches, but collectively referred to as the OpenFlow switch. In addition, the counter is used to carry out statistics on the basic data of the data stream, and the instruction shows the next operation of the data packet which matched with the flow table. The secure channel is the interface of the OpenFlow switch and the controller. The controller can configure and manage the OpenFlow switch according to the format of the OpenFlow protocol.

When a packet arrives at the OpenFlow switch, the information of packet header is extracted and is used to match the flow table item, if the flow table item which matching the packet exists in the middle of the packet flow of OpenFlow, the whole or the part packet will be forwarded to the controller and the controller decides how to process the packet. If the packet is successfully matched to the flow table item in the switch, the OpenFlow switch will forward the packet according to the content of the operation field of the match flow table item.

There are two types of flow table: linear table and hash table. When a flow table item is inserted into the OpenFlow switches, if the 12 tuple information which is used to match the packet contains a determined value, then the flow table item will be inserted into the hash table firstly, if the hash table is full, the flow table item will be inserted into the linear table. However, if the certain fields of the 12 tuple information are provided in the wildcard way, then the flow table item will be only inserted into the linear table. 


\section{Test of OpenFlow Switch}

\section{Measurement Platform.}

In order to test the performance of the OpenFlow switch, Fluke ETHERSCOPE II is used to generate the data flow to meet the high speed and real data flow require of that openflow switch, ES II is in the closed loop of the flow generator and the receiver, also the switch is directly connected with the controller. It is shown as Fig.2.

ES II

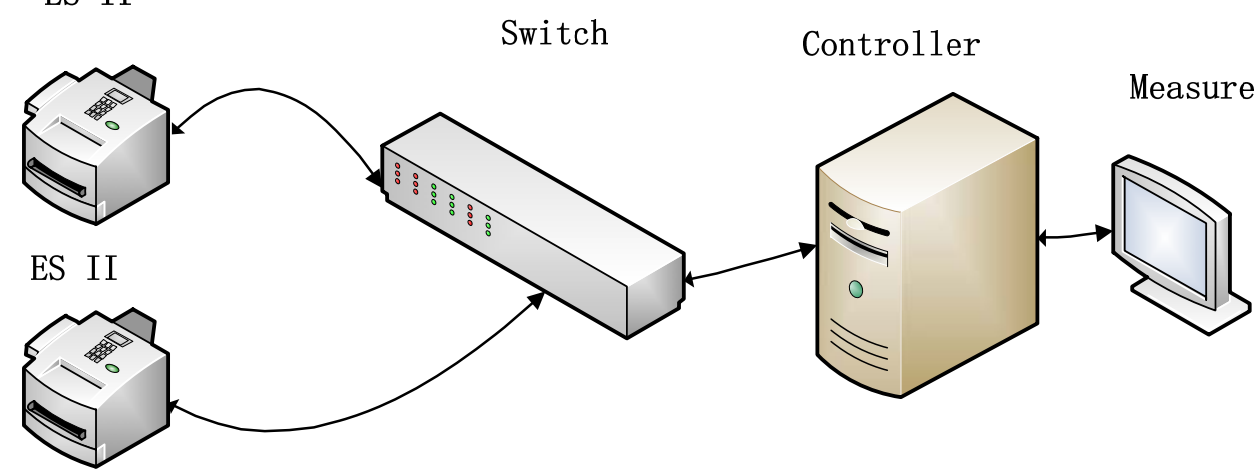

Fig.2 Structure of Measurement Platform

The OpenFlow switch in the experiment is based on Linux platform OpenFlow1.0.0 version. In this version of the switch, there is a linear table and two hash tables, the linear form of the flow table capacity is 100 and the flow of each hash table capacity is $64 \mathrm{~K}$. In the experiment of measuring throughput, in order to obtain the performance of the openflow switch under maximum pressure, the linear table and $128 \mathrm{~K}$ hash table of the openflow switch is all filled up before the throughput experiment, thus the packet arrival openflow switch can directly query flow table and in accordance with the forwarding rules, without a request to the controller of NOX.

After receiving the data of ES II, the OpenFlow Switch forwards the packets, and interact the controller through the port. NOX controller operating measurement module based on Python which intends to measure the interaction of the measurement openflow switch and controller. Through the configuration of the ES II, so as to produce packet header 12 tuple information which can be mapped to a matching rule of the $128 \mathrm{~K}+100$ openflow switches flow table. ES II itself has a function of measuring the performance parameters of real-time packets rate, throughput and packet loss rate, so some parameters of the test can be obtained by ES II.

\section{Experiments.}

\section{(1)TCP/UDP Load Experiment ${ }^{[10]}$}

ES II sending the length of 1480Byte TCP/UDP packets to the switch, then after receiving the packets, the OpenFlow switch forwarding the data packets to the receiver ES II according to the pre insertion flow table rules. The test result is shown in Figure 3. The abscissa is the throughput of the send of ES II; the longitudinal coordinate is the switch throughput and the loss rate.

Using the UDP as the example, when the UDP packet load rate is under 500Mbit/s, the OpenFlow switch of TCP/UDP throughput is almost the same as the load rate, also the packet loss rate is very small, and the time-delay is almost zero; when the TCP/UDP packet load press is more than $500 \mathrm{Mbit} / \mathrm{s}$, lead to a large number of UDP datagrams in the OpenFlow switch buffer queue and eventually lead to overflow of opposites and are discarded. 

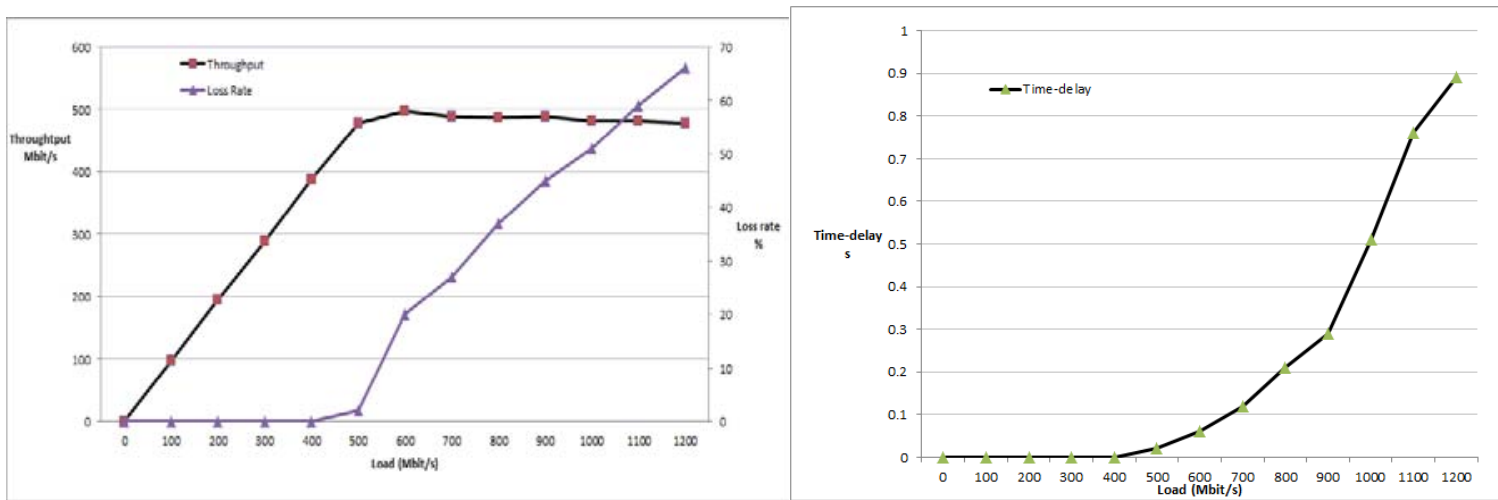

Fig.3 Throughput, Packet loss rate and time-delay of Load experiment

\section{(2)Packets size experiment}

In the experiment, the effects of packet length on OpenFlow switch throughput, packet loss rate and time-delay are studied by transmission of UDP packets with different packet lengths. The experiments are divided into five groups, from the $100 \mathrm{Mbit} / \mathrm{s}-520 \mathrm{Mbit} / \mathrm{s}$, each group increasing length of message between the 100-1480Byte, a total of 14 kinds of tests, and each test duration $60 \mathrm{~s}$, measurement of the OpenFlow switches in each packet size of throughput, packet loss rate and Time-delay. The result of 520Mbit/s UDP load experiment is shown in Figure.4.

It can be found that with the incensement of the length of the packet, the throughput of the OpenFlow switch is increased, and the packet loss rate, the time-delay are reduced.
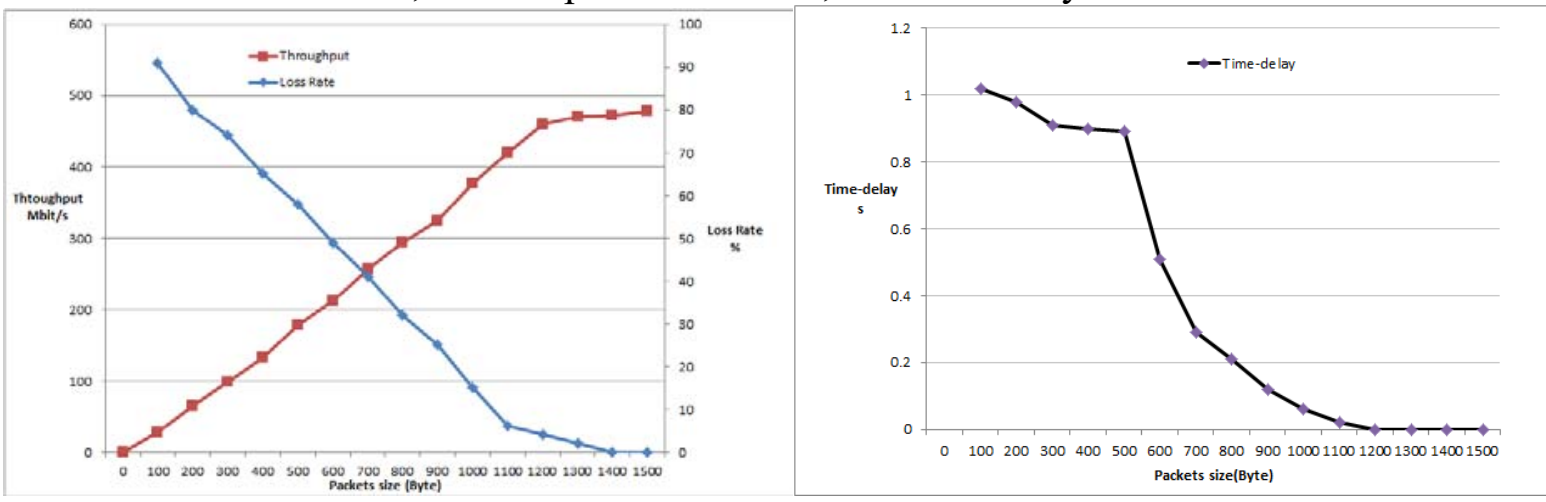

Fig.4 Throughput, Packet loss rate and time-delay of Packets size experiment

\section{(3)The transmission packet quantity experiment}

The mechanism of control plane and data plane separation in OpenFlow switch determines that there is a state of uncertainty between the OpenFlow switch and the controller. Before the flow table packet is inserted into the OpenFlow switch, there might be one similar packet arrival the OpenFlow switch, at this time, these transforming packets will forward as the unknown flow identity to the controller. If the controller does not have a certain fault tolerance, there may be a network failure, and the number of these packets will be greater pressure on the controller and the network, it will affect their work efficiency. Especially, for the UDP transport protocol, because there is no "three handshakes" connection establishment procedure, resulting in the efficiency of the processing of the creating flow table is lower than that of TCP and the corresponding procedure. For example, in terms of the large flow of multimedia data transmission, too much of the UDP packets in the transmission may be submerged controller.

In the experiment, the flow table in OpenFlow switch will not be inserted into any flow table in advance, so when a packet arrives at the switch, it will forward to the NOX controller. NOX controller using Measure module to deal with OpenFlow switch packet, and in accordance with the forwarding strategy, the flow table entries will be inserted to the OpenFlow switch, so that the packet will have the same 12 tuple header information packet and then transmitting to the receiver.

In the experiment, 520Mbit/s UDP packet is generated by ES II, and also the 12 tuple header information of the UDP packet must be the same, 14 sets of measurements according to the length of the UDP packet changes between the 100-1480 Bytes, the duration of each measuring is 60 seconds. 
The measurement component of NOX controller statistical analysis the same UDP packets which repeat arrival, and then getting the number of UDP packet transmission. At the same time using the ES II function to test its throughput, time-delay and packet loss rate. The results are shown in Figure.5.
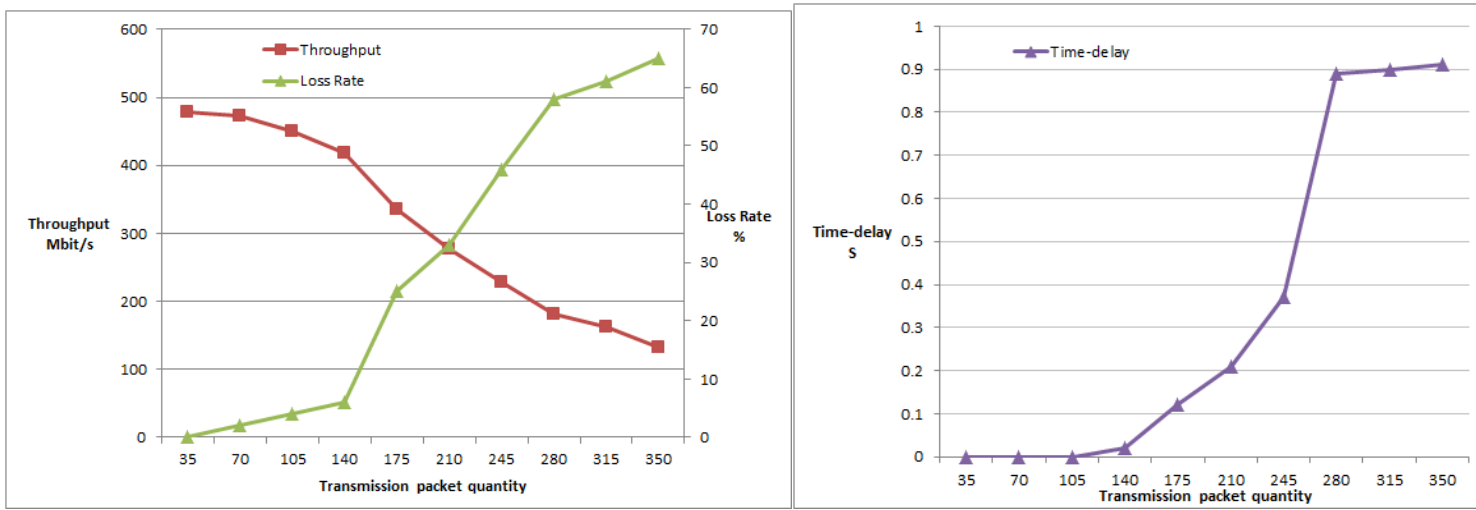

Fig.5 Throughput, Packet loss rate and time-delay of transmission packet quantity experiment

\section{Conclusions}

The main influence parameters of the performance of SDN are CPU performance, SDN version, load of network, packet size and the transforming packet quantity, and the related performance parameters include throughput, time-delay and packet loss rate. It is shown as Table 1.

Table 1 parameters of model of SDN

\begin{tabular}{|c|c|c|}
\hline \multirow{4}{*}{ Influencing Parameters } & Parameters & Controllable \\
\cline { 2 - 3 } & CPU Performance & Not \\
\cline { 2 - 3 } & SDN Version & Not \\
\cline { 2 - 3 } & Load & Yes \\
\cline { 2 - 3 } & packet size & Yes \\
\hline \multirow{3}{*}{ Parameters of Model } & \multicolumn{2}{|c|}{ Throughput } \\
\cline { 2 - 3 } & \multicolumn{2}{|c|}{ Packet Loss Rate } \\
\cline { 2 - 3 } & \multicolumn{2}{|c|}{} \\
\cline { 2 - 3 }
\end{tabular}

The test experiment shows, (1) The OpenFlow switch of TCP/UDP throughput is almost the same as the load rate, when TCP/UDP packet load press is more than the limit, lead to the throughput no longer increase; (2) With the incensement of the size of the packet, the throughput of the OpenFlow switch is increased, and the packet loss rate, the time-delay are reduced; (3) With the incensement of the transmission packet quantity, the throughput of the OpenFlow switch is reduced, and the packet loss rate, the time-delay are increased. The study shows the performance parameters of the SDN are load, packet size and the transforming packet quantity.

The test is the basic and key experiment for establish the performance model of SDN. The research study the SDN OpenFlow switch and find out the main parameters of OpenFlow Swich, and overcome the disadvantages of less parameters of current model. In particular, the transmission packet quantity test method is designed, and influence of the transmission packet quantity is proposed in this paper firstly.

\section{Acknowledgment}

This work was supported by Jiangsu Future Networks Innovation Institute Prospective Research Project on Future Networks.

\section{References}

[1] Network Innovation through OpenFlow and SDN: Principles and Design[M]. CRC Press, 2014. 
[2] Kobayashi M, Seetharaman S, Parulkar G, et al. Maturing of OpenFlow and Software-defined Networking through deployments[J]. Computer Networks, 2014, 61: 151-175.

[3] Song H. Protocol-oblivious forwarding: Unleash the power of SDN through a future-proof forwarding plane[C]/Proceedings of the second ACM SIGCOMM workshop on Hot topics in software defined networking. ACM, 2013: 127-132.

[4] Shirazipour M, John W, Kempf J, et al. Realizing packet-optical integration with SDN and OpenFlow 1.1 extensions[C]//Communications (ICC), 2012 IEEE International Conference on. IEEE, 2012: 6633-6637.

[5] Kotronis V, Dimitropoulos X, Ager B. Outsourcing the routing control logic: Better Internet routing based on SDN principles[C]//Proceedings of the 11th ACM Workshop on Hot Topics in Networks. ACM, 2012: 55-60.

[6] Son S, Shin S, Yegneswaran V, et al. Model checking invariant security properties in OpenFlow[C]//Communications (ICC), 2013 IEEE International Conference on. IEEE, 2013: 1974-1979.

[7] Jarschel M, Oechsner S, Schlosser D, et al. Modeling and performance evaluation of an OpenFlow architecture[C]/Proceedings of the $23 \mathrm{rd}$ international teletraffic congress. International Teletraffic Congress, 2011: 1-7.

[8] Dong L, Li H, He N, et al. Testing OpenFlow interaction property based on hierarchy CPN[C]//Network Protocols (ICNP), 2013 21st IEEE International Conference on. IEEE, 2013: 1-2.

[9] Bianco A, Birke R, Giraudo L, et al. Openflow switching: Data plane performance[C]//Communications (ICC), 2010 IEEE International Conference on. IEEE, 2010: 1-5.

[10] Huang D Y, Yocum K, Snoeren A C. High-fidelity switch models for software-defined network emulation[C]//Proceedings of the second ACM SIGCOMM workshop on Hot topics in software defined networking. ACM, 2013: 43-48 